\title{
BIOSYSTEM WITH 16 HIGHLY-SELECTIVE OPTICAL-CHANNELS FOR BIOLOGICAL FLUIDS ANALYSIS IN THE VISIBLE SPECTRUM
}

\author{
G. Minas ${ }^{(1)}$, J. C. Ribeiro ${ }^{(1)}$, S. Lanceros-Mendez ${ }^{(2)}$, F. Vaz ${ }^{(2)}$, R. F. Wolffenbuttel ${ }^{(3)}$, and J. H. Correia ${ }^{(1)}$ \\ (1) University of Minho, Dept. of Industrial Electronics, \\ ${ }^{(2)}$ University of Minho, Dept. of Physics, \\ ${ }^{(3)}$ Delft University of Technology, Fac. ITS Dept. Microelectronics.
}

\begin{abstract}
This paper reports a biosystem (biological system) for analyzing biochemical substances in biological fluids. Rather than one channel, it comprises 16 highly-selective optical-channels that enable the measurement of the concentration of 16 different biochemical substances. An array of 16-optical filters with high reflectivity and low absorption loss has been designed using dielectric films. Each filter is sensitive in a single wavelength with a FWHM (Full-Width-Half-Maximum) $<5 \mathrm{~nm}$ and peak intensity $>52 \%$. A commercially available band-pass optical filter with a band-pass wavelength in $450-600 \mathrm{~nm}$ is used to enable the use of a white light source for biosystem illumination. Preliminary tests with a single-channel are successfully demonstrated.
\end{abstract}

\section{INTRODUCTION}

Over the past decade, the miniaturization of fluidic analyses systems has become a highly visible and dominant trend in physical and biological sciences [1]. Presently, disease prevention and treatment is often based on the measurement of chemical parameters in biological samples, such as blood, urine, serum, cerebrospinal fluid and plasma. Some of those measurements are performed by optical absorption [2]. However, in most cases, the samples need to be sent to a central laboratory for analysis, and the results of routine tests become available after several hours or even days. The need for rapid and on-line measurements at low concentrations led to the development of biosystems with the fluidic, detection and readout systems integrated in a single-chip [3]. The advantages associated with shrinking clinical analysis systems include improved efficiency with respect to sample size, response times, analytical performance, integration, automation, laboratory safety, throughput and costs. In system signal processing and data pre-handling, the small size and low power consumption of such highly integrated biosystems could make them highly portable and thus suitable for in-situ measurements tasks.

The application of the particular biosystem presented here is the measurement of the concentration, by optical absorption, of 16 different biochemical substances in human's fluids.

\section{BIOSYSTEM DESIGN}

The starting point for the design of the biosystem was macroscopic measurements of the concentration of 16-biochemical substances in well-known standards (Table 1 [2]). These measurements were performed in a $1 \mathrm{~cm}$ lightpath cuvette with a model UV-3101PC SHIMADZU spectrophotometer. The following conclusions can be drawn from these measurements: (1) the intensity of the color produced by the solutions is directly proportional to the concentration of the biochemical substances; (2) each solution presents a linear behavior within the interest concentration range; (3) the absorption spectra of each solution shows a maximum peak at a specific wavelength, described in the third column of Table 1, with FWHMs about $70 \mathrm{~nm}$ [4].

Table 1. The 16-biochemical substances that can be analyzed in the biosystem. U(Urine), S (Serum), CSF (cerebrospinal fluid), and $P$ (Plasma) [2].

\begin{tabular}{|l|c|c|}
\hline $\begin{array}{c}\text { Biochemical } \\
\text { substance }\end{array}$ & $\begin{array}{c}\text { Biological } \\
\text { Fluid }\end{array}$ & $\begin{array}{c}\text { Absorption spectra } \\
\text { maximum peak (nm) }\end{array}$ \\
\hline 17-Ketosteroids & $\mathrm{U}$ & 478 \\
\hline Aldolase & $\mathrm{S}$ & 484 \\
\hline Uric Acid & $\mathrm{U}, \mathrm{CSF}$ & 497 \\
\hline Cholesterol & $\mathrm{S}$ & 500 \\
\hline Glucose & $\mathrm{S}$ & 504 \\
\hline $\begin{array}{l}\text { Glutamic oxalacetic } \\
\text { pyruvic transaminase }\end{array}$ & $\mathrm{S}, \mathrm{P}, \mathrm{CSF}$ & 508 \\
\hline Urea nitrogen & $\mathrm{U}, \mathrm{S}, \mathrm{P}$ & 514 \\
\hline Magnesium & $\mathrm{S}$ & 518 \\
\hline Creatinine & $\mathrm{U}, \mathrm{S}, \mathrm{P}$ & 523 \\
\hline Bile acids & $\mathrm{S}$ & 528 \\
\hline Blood urea nitrogen & $\mathrm{S}, \mathrm{P}$ & 535 \\
\hline Salicylate & $\mathrm{S}$ & 540 \\
\hline Hemoglobin & $\mathrm{P}$ & 543 \\
\hline B Glucuronidase & $\mathrm{S}, \mathrm{U}$ & 548 \\
\hline Bilirubin & $\mathrm{S}$ & 558 \\
\hline Leucine & $\mathrm{U}$ & 567 \\
\hline aminopeptidase & & \\
\hline
\end{tabular}

The biosystem is composed of a glass die that contains the fluidic channels, including the optical filters, and a silicon die that contains the photodetectors and readout electronics. Figure 1 shows schematically the cross-section structure of the biosystem for an individual optical-channel. 


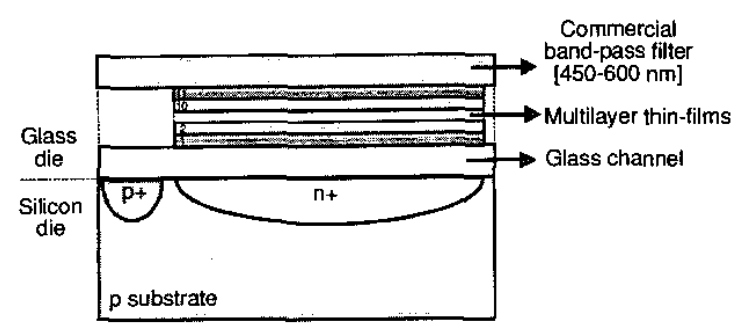

Figure 1. Schematic structure of the biosystem for an individual optical-channel in cross-section.

\section{Glass die}

The glass die is composed of two glass $500 \mu \mathrm{m}$ thick wafers (Figure 2). The first one has the holes for the injection and removing of the liquids (inlets and outlets) and the second one includes the channels. The die comprises three channels with the width of $1 \mathrm{~mm}$ and $6 \mathrm{~mm}$ long. Channel $\mathrm{A}$ is needed to obtain the baseline reference and to calibrate the light source. Channel B allows the analysis of the mixed solution (reagent plus sample). Channel $\mathrm{C}$ is needed to calibrate the biochemical substance concentration (well-known biochemical calibrator). On top of the first glass wafer, the optical filters, schematically shown in Figure 3, are sputtered.

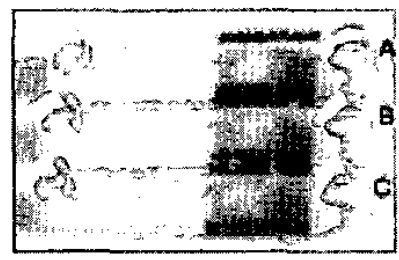

Figure 2. The complete biosystem. It comprises three fluidic channels $(A, B$ and $C)$. The CMOS die is under the channels.

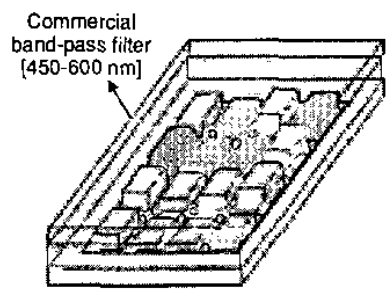

Figure 3. An artist impression of the 16-optical filters (4x4 array) and the commercial band-pass filter.

The device operation is based on optical absorption in a well-defined wavelength of the visible spectrum. The impinging spectrum is filtered, by the optical filters, to a single wavelength, and the intensity of the selected spectral component transmitted through the mixture is measured using an underlying photodetectors array. Thus, each optical-channel is composed by an optical filter with an optical detector underneath. Rather than just one channel [3], this biosystem comprises 16 optical-channels that are tuned for a specific wavelength (see Table 1), which enables the measurement of the concentration of the 16 different biochemical substances.

Each optical filter is composed of a multilayer stack of $\mathrm{TiO}_{2}$ and $\mathrm{SiO}_{2}$ thin-films. The thickness of the layers determines the tuned wavelength. The optical filters use dielectric films which, when properly designed and fabricated, feature high reflectivity and low absorption loss. These high-performance is crucial when the optical filters must provide high transmittance at the tuned wavelength, and low FWHM $(<5 \mathrm{~nm})$. The wavelengths at which the analyzed biochemical substances have their maximum of absorbance are very close, for example, $497 \mathrm{~nm}$ and $500 \mathrm{~nm}$ for the analysis of the uric acid and the cholesterol concentration, respectively (see Table 1). This is the reason the optical filter must be high-selective. Otherwise, the measured absorbance of a certain substance concentration could be affected by its neighborhood, once they have overlapping absorbance spectra (from macroscopic measurements, FWHM $\approx 70 \mathrm{~nm}$ ).

$\mathrm{SiO}_{2}$ have been selected because the dependence of its refractive index on the wavelength for $450 \mathrm{~nm}$ to $600 \mathrm{~nm}$ is almost constant ( 1.465 to 1.457 , respectively). $\mathrm{TiO}_{2}$ have been selected due to fabrication constraints (the sputtering process is well-characterized [5]).

A thin-film optics software package (TFCalc 3.4) was used for the structural optimization of the optical filters. Simulation results show that a multilayer stack (11 layers) of $\mathrm{SiO}_{2} / \mathrm{TiO}_{2}$ thin-films (see Table 2) is the best option for the optical filters in terms of optical characteristics and feasibility. Figure 4 presents the simulated transmittances between $440 \mathrm{~nm}$ and $610 \mathrm{~nm}$ for all the 16 optical-channels, with the layers stack described in Table 2. These results show that each of the channels is sensitive to a single spectral band, with FWHM $<5 \mathrm{~nm}$. Furthermore, the ratio between the transmitted peak and the baseline is larger than 10 and with transmittances higher then $52 \%$. The filters can be easily tuned to cover different spectral bands by adjusting the thickness of one or more layers without affecting the biosystem layout. The performance of the optical filters could be increased by the increase in the layers number, but the fabrication process complexity will increase.

Table 2. Materials, thickness and sputtering sequence of each layer stack for the 16-optical filters array.

\begin{tabular}{|c|c|c|c|c|c|c|c|c|c|c|c|c|c|c|c|c|c|}
\hline \multirow{2}{*}{\multicolumn{2}{|c|}{ Filter number }} & 1 & $\overline{2}$ & 3 & 4 & 5 & $\overline{6}$ & 7 & 8 & 9 & 10 & 11 & 12 & 13 & 14 & 15 & 16 \\
\hline & & \multicolumn{16}{|c|}{$\mathrm{ks}$ (nm) } \\
\hline \multirow{2}{*}{\begin{tabular}{|l} 
Material \\
\end{tabular}} & Peaks & 478 & 4884 & 4497 & 500 & 504 & 508 & 514 & 518 & 523 & 528 & 535 & 540 & 543 & 548 & 558 & 567 \\
\hline & Layer & & & & & & Thict & kness & 5 of 82 & achlas & $y=r(n \pi$ & & & & & & \\
\hline $\mathrm{TiO}_{2}$ & 7 & 285 & 220 & 220 & 220 & 220 & 220 & 220 & 285 & 220 & 220 & \begin{tabular}{|l|}
220 \\
\end{tabular} & 220 & 220 & 220 & 220 & 20 \\
\hline $\mathrm{SiO}_{2}$ & 2 & 260 & 260 & 260 & 260 & 260 & 260 & 260 & 260 & 260 & 260 & 260 & 260 & 260 & 260 & 260 & 260 \\
\hline $\mathrm{TiO}_{2}$ & 3 & 300 & 120 & 180 & 120 & 120 & 120 & 120 & 120 & 120 & 120 & 120 & 505 & 330 & 165 & 165 & 165 \\
\hline $\mathrm{SiO}_{2}$ & 4 & 260 & 260 & 260 & 260 & 260 & 260 & 260 & 260 & 260 & 260 & 260 & 260 & 260 & 260 & 260 & 260 \\
\hline $\mathrm{TiO}_{2}$ & 5 & 120 & 120 & 120 & 120 & 120 & 120 & \begin{tabular}{|l|}
120 \\
\end{tabular} & 120 & 120 & 120 & \begin{tabular}{|l|}
120 \\
\end{tabular} & 120 & 120 & 120 & \begin{tabular}{|l|l|}
120 \\
\end{tabular} & 120 \\
\hline $\mathrm{SiO}_{2}$ & 6 & 220 & 320 & 505 & 505 & 515 & 350 & 360 & 545 & 555 & 385 & \begin{tabular}{|l|}
395 \\
\end{tabular} & 220 & 220 & 220 & 240 & 260 \\
\hline $\mathrm{TiO}_{2}$ & 7 & 125 & 125 & 125 & 125 & 125 & 125 & 125 & 125 & 125 & 125 & \begin{tabular}{|l|}
125 \\
\end{tabular} & 125 & 125 & 125 & 125 & \begin{tabular}{|l|l|}
25 \\
\end{tabular} \\
\hline $\mathrm{SiO}_{2}$ & $\overline{8}$ & 100 & 100 & 100 & 100 & 100 & 100 & 100 & 100 & 100 & 100 & \begin{tabular}{|l|l|}
100 \\
\end{tabular} & 100 & 100 & 100 & 100 & 100 \\
\hline $\mathrm{TiO}_{2}$ & 9 & 140 & 140 & 140 & 140 & 140 & 140 & \begin{tabular}{|l|l|}
140 \\
\end{tabular} & 140 & 140 & 140 & \begin{tabular}{|l|l|}
140 \\
\end{tabular} & 140 & 140 & 140 & 140 & 140 \\
\hline $\mathrm{SiO}$ & 10 & 100 & 150 & 100 & 100 & 100 & 100 & \begin{tabular}{|l|l|}
100 \\
\end{tabular} & 100 & 100 & 100 & \begin{tabular}{|l|l|}
150 \\
\end{tabular} & 100 & 100 & 100 & 100 & 100 \\
\hline TiO & 11 & 140 & 140 & 140 & 140 & $=$ & 140 & 140 & 140 & 140 & 140 & 140 & 140 & 140 & 140 & & 40 \\
\hline
\end{tabular}

TRANSDUCERS ' 03 


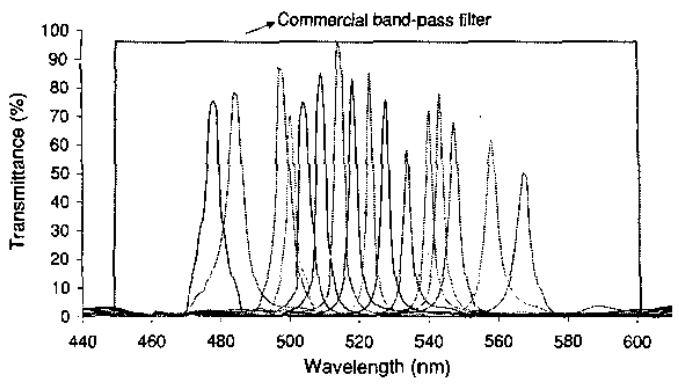

Figure 4. Simulated transmittance vs. wavelength for the 16-optical filters array.

\section{CMOS die}

The photodetectors and the readout electronics are integrated in the same die (Figure 5). The photodetectors are fabricated using the layers available in a CMOS standard process, without additional masks or steps. The photodetectors are pn-junction photodiodes fabricated using a p-substrate/n-diffusion junction (see Figure 1). This structure is chosen because it has the higher quantum efficiency in the desired spectral range, as compared to different types of photodiodes available in a CMOS standard process.

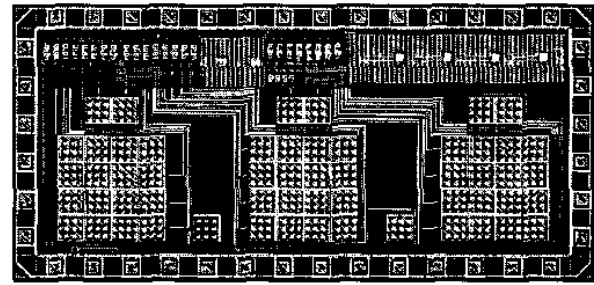

Figure 5. Layout of the CMOS die. Each photodetector has an active area of $200 \times 200 \mathrm{~cm}^{2}$.

It is desirable to integrate the analog to digital conversion with the light sensors. The $A / D$ conversion is performed by using a one-bit first-order sigma delta modulator. A block diagram of the light sensors with the A/D conversion is shown in Figure 6. The circuit consists of the following sections: a current difference amplifier; an integrator and a 1-bit A/D converter in the forward path; and a 1-bit D/A converter in the feedback path of a single feedback loop system. The 1-bit A/D converter is a latched comparator that converts an analog signal into either a high or a low level. Therefore, the biosystem has a bit stream output and allows its use in small data-acquisition and control systems. That bit stream is proportional to the light intensity transmitted through the mixture.

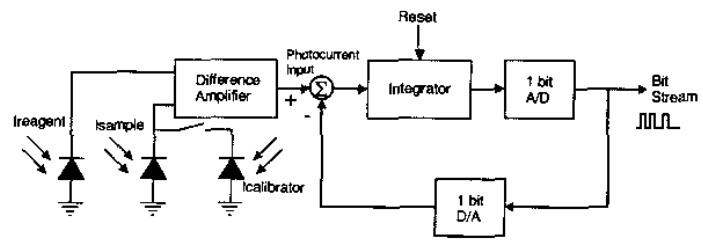

Figure 6. Block diagram of the readout circuit.

\section{BIOSYSTEM FABRICATION}

\section{Glass die}

In the glass die, holes and channels are drilled by using a CNC machine, and then both wafers are glued. Glass is chosen due to its visible transparency and because it is a good insulator.

The optical filters are sputtered on top of the glass die. Films are sputtered by reactive dc magnetron sputtering from high purity $\mathrm{Ti}$ and $\mathrm{Si}$ target. The depositions are carried out in an $\mathrm{Ar} / \mathrm{O}_{2}$ atmosphere in a "home-made" apparatus. The system consists of two vertically opposed rectangular unbalanced magnetrons (type 2 [6]), in a closed field configuration. Prior to depositions, the substrates were ex-situ ultrasonically cleaned. Aluminum is used as a masking material during sputtering because of the high temperature reactive dc magnetron sputtering process. The filter fabrication starts with the sputtering of a $220 \mathrm{~nm}$ thick $\mathrm{TiO}_{2}$ layer over the entire array (see Table 2). In subsequent sputtering steps (for which an aluminum mask is used, Figure 7), the total thickness of the sputtered $\mathrm{TiO}_{2}$ is increased from $220 \mathrm{~nm}$ to $285 \mathrm{~nm}$ (see Table 2). The 16-optical filters are fabricated in 34 masked sputter steps. The aluminum masks are rotated and/or vertically flipped during the fabrication process (see Table 2, Figure 7). This scheme of rotating and/or vertically flipping the masks during the sputtering steps allows the use of only 4 masks. The sputtering steps number could be reduced by increasing the number of the masks.
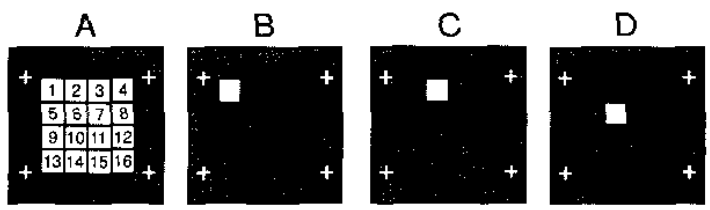

Figure 7. The 4-aluminum masks used to sputter the 11 layers for each optical filter. Masks $B$ and $D$ are rotated to perform filters 1 , $4,16,13$ and $6,7,11,10$, respectively. Mask $C$ is rotated and vertically flipped performing filters $2,8,15,9,3,12,14$, and 5 . The crosses are alignment masks.

\section{CMOS die}

The CMOS compatible photodetectors and readout electronics of the colorimetric detection system are fabricated through a double-metal, single-polysilicon, $1.6 \mu \mathrm{m}$ n-well CMOS standard process. Each photodiode has an active area of $200 \times 200 \mu^{2}$ (see Figure 5). The glass die is glued to the CMOS chip. On top of the biosystem a commercially available band-pass optical filter with a band-pass wavelength in $450-600 \mathrm{~nm}$ is used to enable the use of a white light source for biosystem illumination.

\section{EXPERIMENTAL RESULTS}

The optical spectra and spectral responsivity were measured using a Keithley 487 picoammeter (full-scale range from $10 \mathrm{fA}$ to $2 \mathrm{~mA}$ and a resolution of $5^{1 / 2}$ digit). A 
$200 \mathrm{~W}$ quartz tungsten halogen lamp was used as the white light source for biosystem illumination.

Prior to the fabrication of the 16-optical filters array, system operation was demonstrated on a single-channel. The layers structure of the filter number 3 (see Table 2), designed to be sensitive at the wavelength, $\lambda=497 \mathrm{~nm}$, was sputtered on an empty glass substrate. The reagent used in the measurements was the infinity ${ }^{\mathrm{TM}}$ uric acid reagent from Sigma-Aldrich. It contains approximately $0.5 \mathrm{mmol} / \mathrm{l}$ 4-aminoantipyrine, $1.75 \mathrm{mmol} / 1 \mathrm{TBHB},>32$ Units/l uricase (bacillus sp.), $>1300$ Units/l peroxidase (horseradish), buffer $\mathrm{pH} 8.0$, and $0.05 \%$ sodium azide as preservative [2]. This reagent reacts with a sample of urine containing uric acid in a 50:1 ratio and produces an absorption maximum at a specific wavelength $(\lambda=497 \mathrm{~nm})$. Measurement results are performed from $5 \mathrm{mg} / \mathrm{dl}$ to $120 \mathrm{mg} / \mathrm{dl}$, comprising the range of normal and usually abnormal values in a human's being (which are from 17 to $67 \mathrm{mg} / \mathrm{dl}$ [7]). Optical spectral measurements on the device (with the integrated photodiode) show (see Figure 8 ) that the single-channel is sensitive to its only single wavelength with a peak intensity from $63 \%$ (reagent without uric acid) to $31 \%$ (sample with $120 \mathrm{mg} / \mathrm{dl}$ of uric acid), and FWHM $=3.2 \mathrm{~nm}$, for all concentrations.

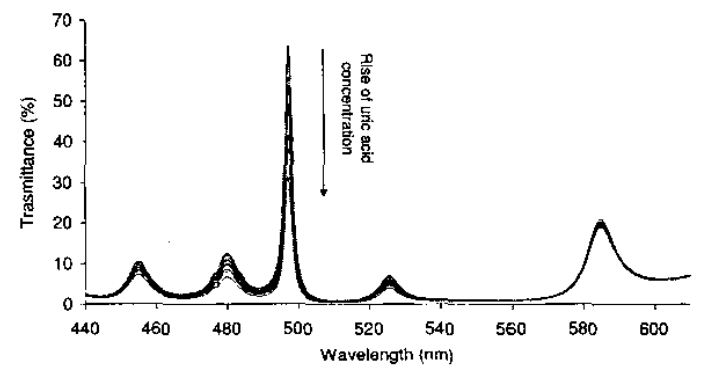

Figure 8. Measured transmittance for a single-channel for different uric acid concentrations $(\lambda=497 \mathrm{~nm})$.

Figure 9 presents the spectral responsivity for the fabricated single-channel, measured with none uric acid concentration. The ratio between the baseline and the peak maximum is 5 . All the measurements are carried out with the commercial band-pass filter showed in Figure 4.

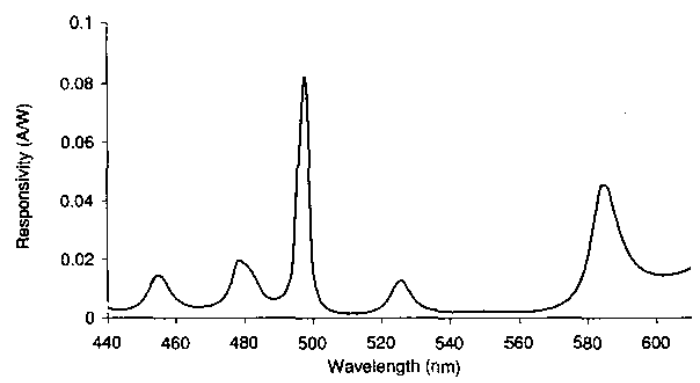

Figure 9. Measured responsivity for a single-channel $(\lambda=497 \mathrm{~nm})$ with no uric acid concentration and a photodetector active area of $200 \times 200 \mathrm{\mu m}^{2}$.

\section{CONCLUSIONS}

A biosystem for analyzing simultaneously the concentration of 16 different biochemical substances in biological fluids is presented in this paper. This performance is obtained with an array of 16-optical filters with high reflectivity and low absorption loss, designed using dielectric films. The biosystem illumination only needs a conventional white light source. This biosystem offers a new approach for clinical analysis, due to the parallel testing for 16 different biochemical substances. The concentration measurement features, in addition, high spectral selectivity, on-line measurement and portability.

\section{ACKNOWLEDGEMENTS}

Support for this research was provided by the Portuguese Foundation of Science and Technology (FCT projects SFRH/BD/1281/2000 and POCTI/33747/ESE/ /1999) and by FEDER.

\section{REFERENCES}

1. S. C. Jakeway, A. J. de Mello, E. Russell, "Miniaturized total analysis systems for biological analysis," Fresenius J. Anal. Chem., 366, pp. 525-539, (2000).

2. "Biochemical and Organic Reagents: for biosciences investigation," Sigma, (2002).

3. Anna J. Tüdõs, Geert A. J. Besselink, Richard B. M. Schasfoort, "Trends in miniaturized total analysis systems for point-of-care testing in clinical chemistry," Lab on a Chip, 1, 83 (2001).

4. G. Minas, J. S. Martins, J. H. Correia, "Highly Selective Optical Detection in a Lab-on-a-Chip for Biological Fluids Analysis," Sensors and Materials, 14-2, pp. 77-89, (2002).

5. E. Ribeiro, A. Malczyk, S. Carvalho, L. Rebouta, J. V. Fernandes, E. Ailves, A. S. Miranda, Surf. Coat. Technol. 515, pp. $151-152$ (2002).

6. B. Window, N. Savides, J. Vac. Sci. Technol. A4 pp. 196, (1986).

7. J. P. Peters, D. D. Van Slyke, "Quantitative Clinical Chemistry Interpretations," Vol. 1, Baltimore, The Williams \& Wilkins Co., (1946). 